This article may be downloaded for personal use only. Any other use requires prior permission of the author and AIP Publishing. This article appeared in Surface Science Spectra, 17(1), 87-92 and may be found at https://doi.org/10.1116/11.20071103.

\section{Gas Phase Deposition of Trichloro(1H,1H,2H,2H-perfluorooctyl)silane on Silicon Dioxide, by XPS}

Michael V. Lee

Brigham Young University, Provo, Utah

Ghaleb Husseini ${ }^{\text {a) }}$

American University of Sharjah, Chemical Engineering, Sharjah, United Arab Emirates

Ken Sautter

Yield Engineering Systems, California

Matthew R. Linford

Brigham Young University, Provo, Utah

(Received 20 November 2007; accepted 17 December 2008; published 7 February 2012)

\begin{tabular}{l}
\hline Accession \#: 01199 \\
Technique: XPS \\
Host Material: poly-(1H,1H,2H,2H- \\
perfluorooctyl)siloxane monolayer \\
on plasma-cleaned silicon \\
Instrument: Kratos Analytical, \\
Manchester, U.K. Axis UltraDLD \\
Major Elements in Spectra: C, O, \\
Si, F \\
Minor Elements in Spectra: P \\
Published Spectra: 5 \\
Spectra in Electronic Record: 5 \\
Spectral Category: technical
\end{tabular}

Monolayers of trichloro $\left(\mathrm{H}, 1 \mathrm{H}, 2 \mathrm{H}, 2 \mathrm{H}\right.$-perfluorooctyl)silane, $\mathrm{Cl}_{3} \mathrm{SiCH}_{2} \mathrm{CH}_{2}\left(\mathrm{CF}_{2}\right)_{5} \mathrm{CF}_{3}$, were deposited via chemical vapor deposition onto the native oxide layer on silicon after plasma-cleaning. The samples have high hydrophobicity, and provide a valuable comparison to perfluorinated alkyl silane layers obtained by liquid deposition. Gas-phase deposition of perfluorinated alkyl silanes is a useful means for reducing stiction in micro- and nano-electromechanical systems, which have narrow spaces that can trap bubbles and prevent liquid-based silane passivation. (C) 2012 American Vacuum Society. [DOI: $10.1116 / 11.20071103]$

Keywords: perfluorinated silane; gas-phase; native silicon dioxide

\section{SPECIMEN DESCRIPTION (ACCESSION \#01199)}

Host Material: poly- $(1 \mathrm{H}, 1 \mathrm{H}, 2 \mathrm{H}, 2 \mathrm{H}-$ perfluorooctyl)siloxane monolayer on plasma-cleaned silicon

\section{CAS Registry \#: 78560-45-9}

Host Material Characteristics: homogeneous; solid; organic compound

Chemical Name: silicon oxide $\left(\mathrm{SiO}_{2}\right)$

Source: Moctco Silicon Technologies, Inc.

Host Composition: monolayer on plasma-cleaned native oxide on silicon

Form: monolayer

Lot \#: W9969

Structure: Si $\left(\begin{array}{lll}1 & 0 & 0\end{array}\right)$

History \& Significance: Silanes are often used to modify the surface free energy and/or add a functional group to silicon/silicon dioxide and/or glass surfaces. Perfluorinated alkyl silanes are desirable because of their hydrophobic natures and favorable tribological properties. The hydrophobicity of the resulting monolayers provides a protective coating that can effectively exclude water and oil. The reduction of static friction on silicon surfaces is especially important in the development of micro- and nano-electromechanical systems (MEMs and NEMs) since stiction has been a leading cause of device failure. Gas phase deposition provides a method for better coverage for critical areas of these devices.

\footnotetext{
a)Author to whom correspondence should be addressed.
}

Silicon wafers with a native oxide layer were cleaned with a 6-min exposure to a $500 \mathrm{~W}$ oxygen plasma in a YES-1224P (Yield Engineering Systems; Livermore, CA, USA) chemical vapor deposition system. After cleaning and without venting, trichloro $(1 \mathrm{H}, 1 \mathrm{H}, 2 \mathrm{H}, 2 \mathrm{H}$-perfluorooctyl)silane was injected. The processing temperature was $100^{\circ} \mathrm{C}$. After cleaning, and without venting, the oven was heated to $100{ }^{\circ} \mathrm{C}$ and trichloro $(1 \mathrm{H}, 1 \mathrm{H}, 2 \mathrm{H}, 2 \mathrm{H}$ perfluorooctyl)silane was injected. After ten minutes of exposure the oven was evacuated, cooled to room temperature, and opened. The deposition was confirmed by measuring the static water contact angle of one sample, which was $110^{\circ}$.

As Received Condition: silicon wafer (DIA 125)

Analyzed Region: three different spots on each sample

Ex Situ Preparation/Mounting: standard Kratos sample holder

In Situ Preparation: none

Pre-Analysis Beam Exposure: total irradiation time per spot $<22$ min

Charge Control: none

Temp. During Analysis: $298 \mathrm{~K}$

Pressure During Analysis: $<6.65 \times 10^{-7} \mathrm{~Pa}$

\section{INSTRUMENT DESCRIPTION}

Manufacturer and Model: Kratos Analytical, Manchester, U.K. Axis UltraDLD

Analyzer Type: Other

Detector: multichannel plates and delay line detector

Number of Detector Elements: 128

Analyzer Description: hemispherical mirror analyser, magnetic snorkel lens 


\section{Spectrometer}

Analyzer Mode: constant pass energy

Throughput $\left(T=E^{N}\right): \mathrm{N}=0$

Excitation Source Window: none

Excitation Source: $\mathrm{Al} K_{\alpha}$, monochromatic

Source Energy: $1486.69 \mathrm{eV}$

Source Strength: $225 \mathrm{~W}$

Source Beam Size: $800 \mu \mathrm{m} \times 800 \mu \mathrm{m}$

Signal Mode: multichannel direct

\section{- Geometry}

Incident Angle: $45^{\circ}$

Source to Analyzer Angle: $45^{\circ}$

Emission Angle: $0^{\circ}$

Specimen Azimuthal Angle: $0^{\circ}$

Acceptance Angle from Analyzer Axis: $0^{\circ}$ Comments:
DATA ANALYSIS METHOD

Peak Shape and Background Method: Shirley background function; in the case where the Shirley function was undefined, a linear background was used.

Quantitation Method: Sensitivity factors were obtained from software supplied by Kratos. The areas are the areas above the background.

\section{ACKNOWLEDGMENTS}

The authors thank Loren Rieth for his assistance in operating the Kratos instrument and in accurately reporting the instrument's characteristics.

\section{REFERENCES}

1. J. Y. Huang, K. J. Song, A. Lagoutchev, P. K. Yang, and T. J. Chuang, Langmuir 13, 58 (1997).

2. Z. Tao and B. Bhushan, Langmuir 21, 2391 (2005).

3. E. Hoque, J. A. DeRose, R. Houriet, P. Hoffman, and H. J. Mathieu, Chem. Mater. 19, 798 (2007). 


\begin{tabular}{|c|c|c|c|c|c|c|c|}
\hline \multicolumn{8}{|c|}{ SPECTRAL FEATURES TABLE } \\
\hline $\begin{array}{l}\text { Spectrum } \\
\text { ID \# }\end{array}$ & $\begin{array}{l}\text { Element/ } \\
\text { Transition }\end{array}$ & $\begin{array}{c}\text { Peak } \\
\text { Energy } \\
(\mathrm{eV})\end{array}$ & $\begin{array}{c}\text { Peak } \\
\text { Width } \\
\text { FWHM } \\
\text { (eV) }\end{array}$ & $\begin{array}{c}\text { Peak } \\
\text { Area } \\
\text { (eV-cts/s) }\end{array}$ & $\begin{array}{c}\text { Sensitivity } \\
\text { Factor }\end{array}$ & $\begin{array}{c}\text { Concentration } \\
\text { (at. \%) }\end{array}$ & $\begin{array}{c}\text { Peak } \\
\text { Assignment }\end{array}$ \\
\hline 01199-02 & $\mathrm{C} 1 \mathrm{~s}$ & 292.1 & 1.423 & 2451 & 0.278 & 6.2 & $\mathrm{C}-\mathrm{F}_{2}$ \\
\hline 01199-02 & C 1s & 285.4 & 1.223 & 1708 & 0.278 & 4.3 & C-C, C-H \\
\hline 01199-02 & $\mathrm{C} 1 \mathrm{~s}$ & 294.5 & 0.892 & 530 & 0.278 & 1.3 & $\mathrm{C}-\mathrm{F}_{3}$ \\
\hline 01199-02 & $\mathrm{C} 1 \mathrm{~s}$ & 286.9 & 1.494 & 1069 & 0.278 & 2.7 & $\mathrm{C}-\mathrm{O}$ \\
\hline 01199-03 & O 1s & 533.2 & 1.506 & 42871 & 0.780 & 34.7 & $\cdots$ \\
\hline 01199-04 & Si $2 p$ & 99.5 & 0.402 & 4821 & 0.328 & 11.7 & Si wafer \\
\hline 01199-04 & Si $2 p$ & 100.1 & 0.563 & 3878 & 0.328 & 9.4 & Si split \\
\hline 01199-04 & Si $2 p$ & 103.7 & 1.663 & 3335 & 0.328 & 8.1 & $\mathrm{SiO}_{2}$ \\
\hline 01199-04 & Si $2 p$ & 101.9 & 1.575 & 1303 & 0.328 & 3.1 & other $\mathrm{Si}$ \\
\hline 01199-05 & F 1s & 689.6 & 1.750 & 26982 & 1.000 & 15.8 & $\mathrm{~F}-\mathrm{C}$ \\
\hline 01199-05 & F 1s & 687.4 & 2.373 & 4634 & 1.000 & 2.7 & $\mathrm{~F}-\mathrm{O}$ \\
\hline
\end{tabular}

\begin{tabular}{|c|c|c|c|c|c|}
\hline \multicolumn{6}{|c|}{ GUIDE TO FIGURES } \\
\hline $\begin{array}{l}\text { Spectrum } \\
\text { (Accession) \# }\end{array}$ & $\begin{array}{l}\text { Spectral } \\
\text { Region }\end{array}$ & $\begin{array}{c}\text { Voltage } \\
\text { Shift* }^{*}\end{array}$ & Multiplier & Baseline & Comment \# \\
\hline 1 & survey & 0 & 1 & 0 & \\
\hline 2 & $\mathrm{C} 1 \mathrm{~s}$ & 0 & 1 & 0 & \\
\hline 3 & $\mathrm{O} 1 \mathrm{~s}$ & 0 & 1 & 0 & \\
\hline 4 & Si $2 p$ & 0 & 1 & 0 & \\
\hline 5 & $\mathrm{~F} 1 \mathrm{~s}$ & 0 & 1 & 0 & \\
\hline
\end{tabular}

* Voltage shift of the archived (as-measured) spectrum relative to the printed figure. The figure reflects the recommended energy scale correction due to a calibration correction, sample charging, flood gun, or other phenomenon. 


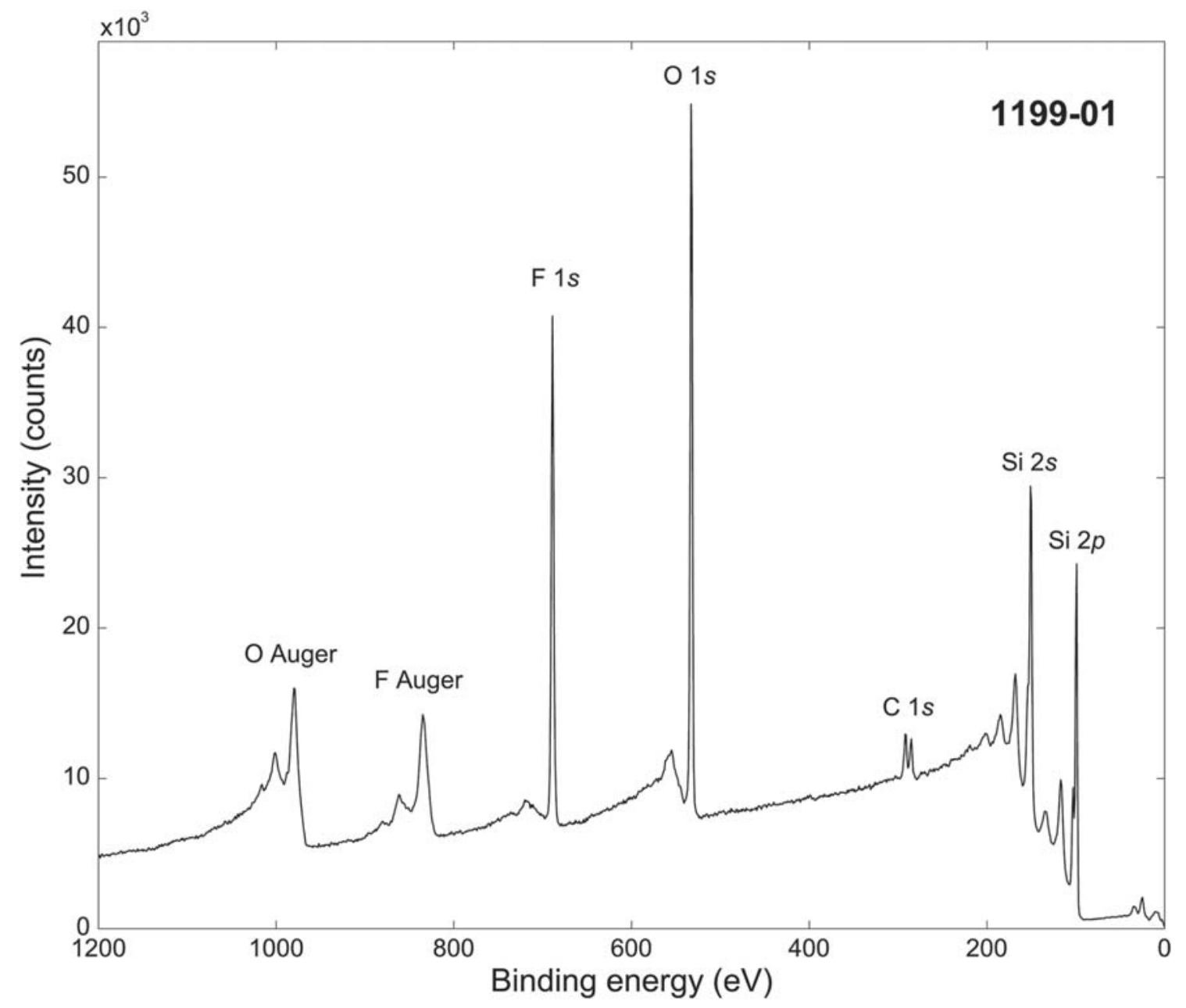

\begin{tabular}{|c|c|}
\hline Accession\# & 01199-01 \\
\hline Host Material & $\begin{array}{c}\text { poly- }(1 \mathrm{H}, 1 \mathrm{H}, 2 \mathrm{H}, 2 \mathrm{H} \text {-perfluorooctyl)siloxane monolayer } \\
\text { on plasma-cleaned silicon }\end{array}$ \\
\hline Technique & XPS \\
\hline Spectral Region & survey \\
\hline Instrument & Kratos Analytical, Manchester, U.K. Axis UltraDLD \\
\hline Excitation Source & Al $K_{\alpha}$ monochromatic \\
\hline Source Energy & $1486.69 \mathrm{eV}$ \\
\hline Source Strength & $225 \mathrm{~W}$ \\
\hline Source Size & $0.8 \mathrm{~mm} \times 0.8 \mathrm{~mm}$ \\
\hline Analyzer Type & hemispherical mirror analyser, magnetic snorkel lens \\
\hline Incident Angle & $45^{\circ}$ \\
\hline Emission Angle & $0^{\circ}$ \\
\hline Analyzer Pass Energy & $150 \mathrm{eV}$ \\
\hline Analyzer Resolution & $1.5 \mathrm{eV}$ \\
\hline Total Signal Accumulation Time & $240 \mathrm{~s}$ \\
\hline Total Elapsed Time & $440 \mathrm{~s}$ \\
\hline Number of Scans & 1 \\
\hline Effective Detector Width & $1200 \mathrm{eV}$ \\
\hline
\end{tabular}




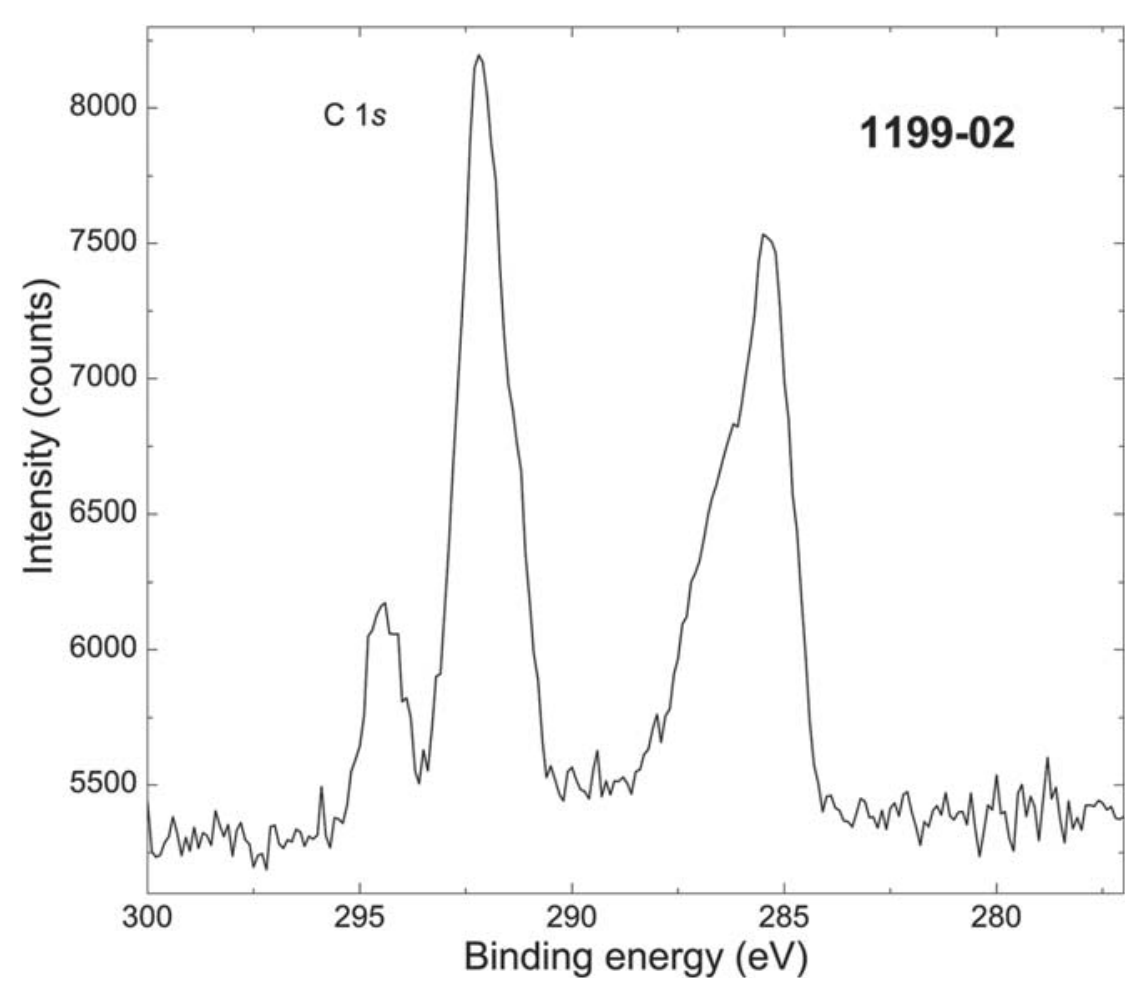

- Accession \#: 01199-02

- Host Material: poly- $(1 \mathrm{H}, 1 \mathrm{H}$, $2 \mathrm{H}, 2 \mathrm{H}$-perfluorooctyl) siloxane monolayer on plasma-cleaned silicon

- Technique: XPS

- Spectral Region: C $1 \mathrm{~s}$

Instrument: Kratos Analytical Manchester, U.K. Axis UltraDLD

Excitation Source: Al $K_{\alpha}$ monochromatic

Source Energy: $1486.69 \mathrm{eV}$

Source Strength: $225 \mathrm{~W}$

Source Size: $0.8 \mathrm{~mm} \times 0.8 \mathrm{~mm}$

Analyzer Type: hemispherical mirror analyser, magnetic snorkel lens

Incident Angle: $45^{\circ}$

Emission Angle: $0^{\circ}$

Analyzer Pass Energy: $50 \mathrm{eV}$

Analyzer Resolution: $0.5 \mathrm{eV}$

Total Signal Accumulation Time: $139 \mathrm{~s}$

Total Elapsed Time: $339 \mathrm{~s}$

Number of Scans: 2

Effective Detector Width: $19.70 \mathrm{eV}$

- Accession \#: 01199-03

- Host Material: poly- $(1 \mathrm{H}, 1 \mathrm{H}$, $2 \mathrm{H}, 2 \mathrm{H}$-perfluorooctyl) siloxane monolayer on plasma-cleaned silicon

- Technique: XPS

- Spectral Region: $01 s$

Instrument: Kratos Analytical, Manchester, U.K. Axis UltraDLD

Excitation Source: Al $K_{\alpha}$ monochromatic

Source Energy: $1486.69 \mathrm{eV}$

Source Strength: $225 \mathrm{~W}$

Source Size: $0.8 \mathrm{~mm} \times 0.8 \mathrm{~mm}$

Analyzer Type: hemispherical mirror analyser, magnetic snorkel lens

Incident Angle: $45^{\circ}$

Emission Angle: $0^{\circ}$

Analyzer Pass Energy: $50 \mathrm{eV}$

Analyzer Resolution: $0.5 \mathrm{eV}$

Total Signal Accumulation Time: $109 \mathrm{~s}$

Total Elapsed Time: $309 \mathrm{~s}$

Number of Scans: 2

Effective Detector Width: $19.70 \mathrm{eV}$ 


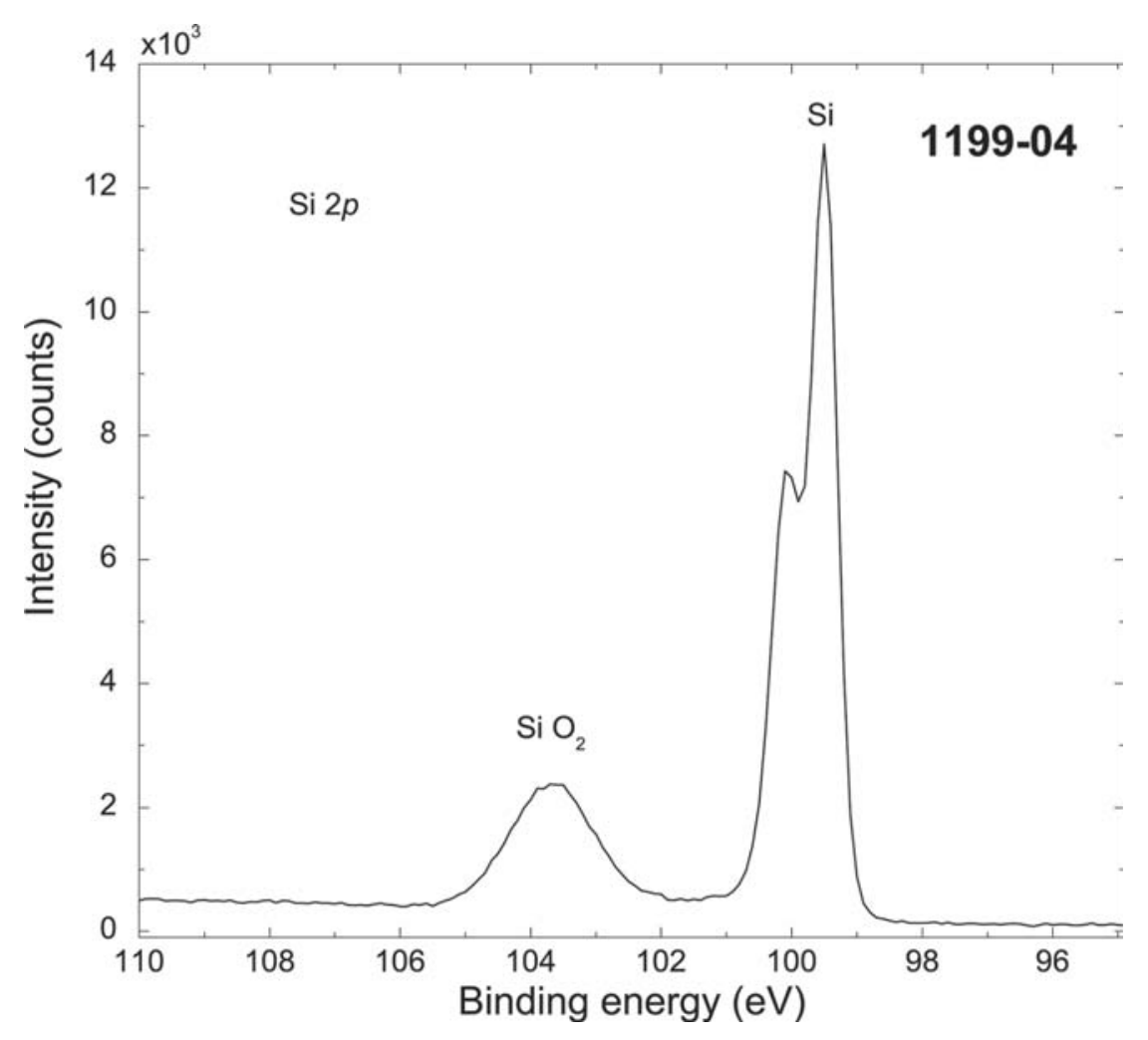

- Accession \#: 01199-04

- Host Material: poly- $(1 \mathrm{H}, 1 \mathrm{H}$, $2 \mathrm{H}, 2 \mathrm{H}$-perfluorooctyl)siloxane monolayer on plasma-cleaned silicon

- Technique: XPS

- Spectral Region: Si $2 p$

Instrument: Kratos Analytical, Manchester, U.K. Axis UltraDLD

Excitation Source: Al $K_{\alpha}$ monochromatic

Source Energy: $1486.69 \mathrm{eV}$

Source Strength: $225 \mathrm{~W}$

Source Size: $0.8 \mathrm{~mm} \times 0.8 \mathrm{~mm}$

Analyzer Type: hemispherical mirror analyser, magnetic snorkel lens Incident Angle: $45^{\circ}$

Emission Angle: 0

Analyzer Pass Energy: $50 \mathrm{eV}$ Analyzer Resolution: $0.5 \mathrm{eV}$ Total Signal Accumulation Time: $91 \mathrm{~s}$ Total Elapsed Time: $291 \mathrm{~s}$

Number of Scans: 2

Effective Detector Width: $19.70 \mathrm{eV}$

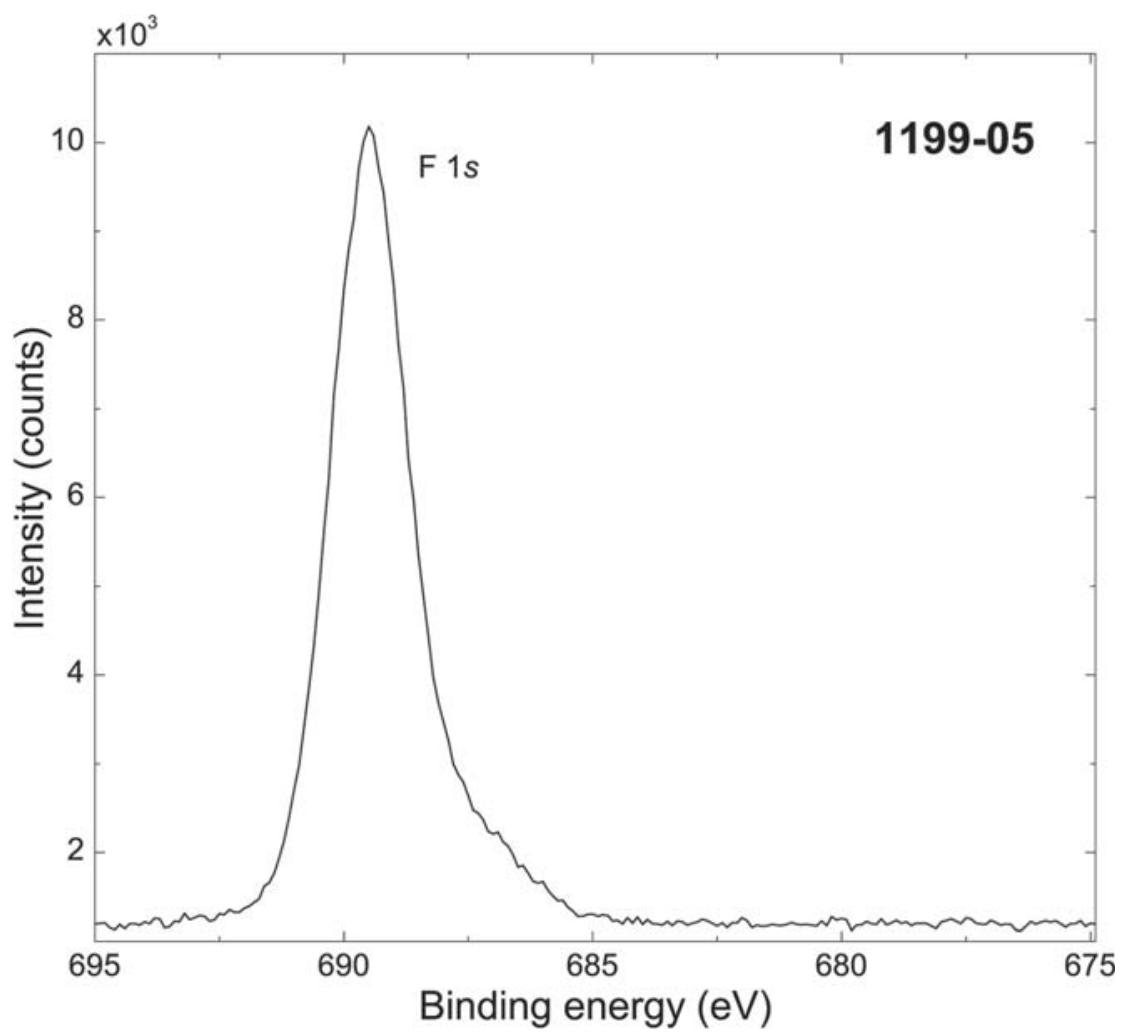

Accession \#: 01199-05

- Host Material: poly- $(1 \mathrm{H}, 1 \mathrm{H}$, $2 \mathrm{H}, 2 \mathrm{H}$-perfluorooctyl)siloxane monolayer on plasma-cleaned silicon

- Technique: XPS

- Spectral Region: F $1 s$

Instrument: Kratos Analytical, Manchester, U.K. Axis UltraDLD

Excitation Source: Al $K_{\alpha}$ monochromatic

Source Energy: $1486.69 \mathrm{eV}$

Source Strength: $225 \mathrm{~W}$

Source Size: $0.8 \mathrm{~mm} \times 0.8 \mathrm{~mm}$

Analyzer Type: hemispherical mirror analyser, magnetic snorkel lens

Incident Angle: $45^{\circ}$

Emission Angle: $0^{\circ}$

Analyzer Pass Energy: $50 \mathrm{eV}$

Analyzer Resolution: $0.5 \mathrm{eV}$

Total Signal Accumulation Time: $121 \mathrm{~s}$ Total Elapsed Time: $321 \mathrm{~s}$

Number of Scans: 2

Effective Detector Width: $19.70 \mathrm{eV}$ 